\title{
Is there hope to treat glioblastoma effectively?
}

\section{CNS Oncology}

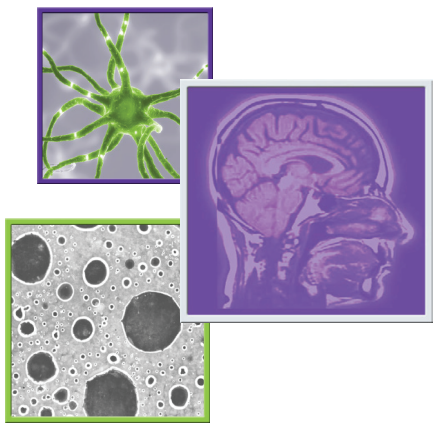

Thomas Mehrling* speaks to Roshaine Gunawardana, Commissioning Editor: Thomas Mehrling was appointed Managing Director of Mundipharma EDO GmbH, Basel, in January 2013 and brings extensive experience with more than 17 years in the industry to this role. During his career, he has held various senior positions in different companies across almost all functions in drug development and commercialization. Most recently, he held the position of International Director Oncology Strategy (2011-2013). From 2004 to 2011 he served as European Director Oncology at Mundipharma International Ltd. During his tenure the oncology business of the European Mundipharma network of independent associated companies was set up and two major products were launched in Europe, DepoCyte ${ }^{\circledast}$ and Levact ${ }^{\oplus}$ (Ribomustin ${ }^{\oplus}$, Treanda ${ }^{\oplus}$ ). He joined Mundipharma in 2000 as Head of Business Development. Prior to Mundipharma, he was Senior Vice President of the global CRO Medical Affairs at Staticon International, and prior to this he acted as Medical Leader at Takeda European $R \& D$ center. Dr Mehrling is a certified Pharmacist with a PhD in pharmacology and a certified Physician trained in haemato-oncology. He obtained his $\mathrm{PhD}$ from Frankfurt University following work on developing a new 5-HT3 antagonist to treat nausea and vomiting and developed a particular interest in mechanisms of multidrug resistance into chemotherapy. Dr Mehrling earned his MD degree through his work in the Department of Internal medicine at Frankfurt University (Hemato-oncology and Cardiology) where he worked for several years before starting his career in the pharmaceutical industry.

\section{Q What led to your interest in specializing in pharmacological science early on in your career?}

The subject found me! Clearly it was the enthusiasm of my teacher at the time that hooked me up too. I was fascinated that body function could be influenced and healed by pharmacologically active substances and to understand how this works, how the drug works on the body and how the body modifies the drug thrilled me. My teacher, Prof Dr Ernst Mutschler in Frankfurt, took us with such an enthusiasm through this subject that I immediately chose to stay with him for my $\mathrm{PhD}$ thesis.

\section{Q As a board certified physician, and also having had experience in the oncology pharmaceuticals industry, what are your thoughts on the relationship between industry and 'on the ground' healthcare? How, if at all, would you like this to change? I think that patients have the greatest benefit where academia and industry work closest together. This is particularly fruitful in early stage drug development. Thinking about HIV, I do not think it would have been possible to develop effective therapy so quickly if this collaboration had not worked out so brilliantly. A similar development is now seen in the CAR-T cell field. The technology came out of academia but the industry is required to make this work alongside regulatory standards, to get these most important}

*Mundipharma EDO GmbH, St. Alban-Rheinweg 74, CH-4020, Basel, Switzerland;

thomas.mehrling@mundipharma-edo.com

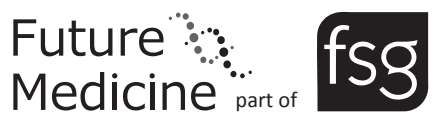


medicines approved. This collaboration is clearly bringing medicines faster to the patients in need and should be intensified. We also need to make sure that concerns about bribery are not overshadowing this symbiosis resulting in a detrimental effect, but rather make an effort to communicate these benefits better to politicians and the public.

\section{Q What are the challenges currently associated with the treatment of human glioblastoma?}

Unlike in other fields of cancer, the GBM remains a nasty tumor with a dismal outcome. This is related to late diagnosis and aggressive proliferation, as well as the fact that the tumor is creeping widely into healthy brain tissue often limiting the impact of surgery. In addition the tumor is resistant to many therapeutics and the drugs which can be used, need to cross the blood-brain barrier, which is often disrupted in the tumor tissue. Some of the tumor biology, such as the MGMT methylation status, also renders standard therapy like temozolomide less or noneffective, and radiotherapy does not seem to have great benefit in elderly patients. Clearly there is huge unmet need and we desperately need better medicines.

Q Mundipharma EDO initially studied the efficacy of EDO-S101 in models of relapsed/ refractory multiple myeloma and other hematological malignancies. What led to investigations into glioblastoma and EDO-S101?

We know that our compound is a potent alkylating agent in addition to the HDAC inhibition properties, and is also lipophilic. This, altogether, is a strong prerequisite to think about treating CNS tumors. We have also seen some data that show that the addition of the HDAC inhibition component could help to overcome some resistance to conventional drugs. We thought this was a great hypothesis and went on to study the drug in preclinical models.

Q Mundipharma EDO recently released data confirming that EDO-S101 is effective in preclinical models of human glioblastoma. Can you please briefly summarize the main results of the study?

We did the relevant and complementary experiments in two different institutions. This gives us some confidence that the drug should be tested in patients. We found in vitro and in vivo that our drug has activity irrespective of MGMT methylation status and works in cells highly resistant to temozolomide. It was also confirmed that the drug crosses the blood-brain barrier and, most importantly, we reach concentrations that are pharmacologically active. There was also clear synergy with radiotherapy, which is still standard of care post-surgery in these patients. We then looked at secondary brain tumors, for example, from breast cancer and primary CNS lymphoma, and found activity. This, all together, makes us think that we should include patients with CNS tumors in our Phase I program.

\section{Q Phase I studies with EDO-S101 are due to start later this year. Should the results continue to be promising, what do you think this would mean for the future of glioblastoma treatment?}

We would hope that EDO-S101 could be of benefit to all patients with GBM and, based on the safety profile, could be established as the preferred combination partner to other forthcoming agents, such as pan-PI3K antagonists in GBM or ibrutinib in primary CNS lymphoma. Ultimately the best option for patients will be with synergistic combinations that target multiple pathways in GBM. We are also hoping that we can look at combinations with PARP- and PI3K-inhibitors as well as immune-oncology drugs in CNS cancers, such as CAR-T cell products. We have initial data suggesting that our drug is reducing inhibitory T-regs and increasing cytotoxic T-cells in the tumor environment.

\section{Q Can you tell our readers about any other} CNS oncology-related projects that you are working on at the moment?

The other programs in our pipeline are antibody drug conjugates. We are in early days but we are exploring several potential indications for these constructs, including GBM. If more powerful cytotoxic agents are brought selectively to the tumor, we may see improved efficacy over and above what we can achieve now. The need for innovation is huge in this disease.

\section{Q How do you see research into CNS cancers} progressing in the next 5-10 years?

We may see improved diagnostics coming along. Deep sequencing and easy testing for circulating tumor DNA may allow for earlier interventions. 
I believe that surgery will be the mainstay of therapy but we hopefully will have a significantly expanded armentarium of drugs available to offer treatment. This may include powerful antibody drug conjugates and immunotherapeutic compounds in addition to small molecules influencing signaling pathways.

Q Do you have any final thoughts for our readers?

Breakthrough therapies are only possible if we work together, academia and industry. But we also need to reach the public and particularly patients and their relatives. We need to effectively communicate that this is the only way to invent new therapies and beat cancer. We need to keep this environment and our image clean by performing proper research and clinical trials. I firmly believe we eventually will succeed and cure cancer or at least make it a chronic disease.

\section{Disclaimer}

The opinions expressed in this interview are those of the interviewee and do not necessarily reflect the views of Future Medicine Ltd.

\section{Financial \& competing interests disclosure}

TMehrling is an employee of Mundipharma EDO GmbH. $T$ Mehrling has no other relevant affliations or financial involvement with any organization or entity with a financial interest in or financial conflict with the subject matter or materials discussed in the manuscript apart from those disclosed.

No writing assistance was utilized in the production of this manuscript. 